\title{
Optimization of 3D chemical imaging by soft X-ray spectro-tomography using a compressed sensing algorithm
}

Juan Wu, ${ }^{1}$ Mirna Lerotic, ${ }^{2}$ Sean Collins, ${ }^{3}$ Rowan Leary, ${ }^{3}$ Zineb Saghi, ${ }^{3}$ Paul Midgley, ${ }^{3}$ Slava Berejnov, ${ }^{4}$ Darija Susac, ${ }^{4}$ Juergen Stumper, ${ }^{4}$ Gurvinder Singh ${ }^{5}$ and Adam P. Hitchcock ${ }^{1}$

1. Dept. of Chemistry \& Chemical Biology, McMaster University, Hamilton, ON, Canada

2. 2nd Look Consulting, Hong Kong

3. Department of Materials Science and Metallurgy, University of Cambridge, Cambridge, UK

4. Automotive Fuel Cell Cooperation (AFCC) Corporation, Burnaby, BC, Canada

5. Department of Materials Science and Engineering, Norwegian University of Science and Technology, Trondheim, Norway

Abstract: Soft X-ray spectro-tomography provides 3D chemical mapping based on natural Xray absorption properties. Since radiation damage is intrinsic to X-ray absorption, it is important to find ways to maximize signal within a given dose. For tomography, using the smallest number of tilt series images that gives a faithful reconstruction is one such method. Compressed sensing (CS) methods have relatively recently been applied to tomographic reconstruction algorithms, providing faithful 3D reconstructions with a much smaller number of projection images than when conventional reconstruction methods are used. Here, CS is applied in the context of scanning transmission X-ray microscopy tomography. Reconstructions by weighted backprojection, the simultaneous iterative reconstruction technique, and CS are compared. The effects of varying tilt angle increment and angular range for the tomographic reconstructions are examined. Optimization of the regularization parameter in the CS reconstruction is explored and discussed. The comparisons show that CS can provide improved reconstruction fidelity relative to weighted back projection and simultaneous iterative reconstruction techniques, with increasingly pronounced advantages as the angular sampling is reduced. In particular, missing wedge artefacts are significantly reduced and there is enhanced recovery of sharp edges. Examples of using CS for low-dose scanning transmission X-ray microscopy spectroscopic tomography are presented.

Keywords: 3D chemical mapping; X-ray tomography; compressed sensing; STXM; electron tomography

Microscopy \& Microanalysis (original submitted 12 Feb 2017; REVISED submitted 06 May 2017; Second revision submitted 26-Jun-17) Tomo-CS.doc Last changed: 26 Jun 2017

SHORT TITLE: Compressed sensing tomography 


\section{Introduction}

Tilt series tomography is now a popular and well-developed method for measuring the three-dimensional (3D) morphology and spatial distribution of materials (Baruchel et al, 2006, Boudin et al, 2010, Leary et al. 2012, Ercius et al. 2015). A typical tilt series tomography data set is composed of a set of $2 \mathrm{D}$ projection images collected at different sample rotation angles. A reconstruction algorithm is then applied to the set of projection images to generate $3 \mathrm{D}$ information. The fidelity of the resulting tomographic reconstruction is related to both the image collection and the reconstruction algorithm.

Scanning Transmission X-ray Microscopy (STXM) (Howells et al. 2007, Ade \& Hitchcock 2008, Hitchcock 2012) provides chemical speciation and high resolution quantitative mapping in projection with a routine resolution of $\sim 30 \mathrm{~nm}$ and a state of the art resolution of 10 nm (Chao et al 2012). STXM spectro-tomography (multi-angle tomographic acquisition at multiple X-ray energies) (Johansson et al. 2006, Johansson et al. 2007, Wang et al. 2011, Schmid et al. 2016) provides 4D information, in the form of chemical mapping in 3D, with a spatial and chemical resolution dependent on sampling and data processing. Near-edge X-ray absorption fine structure (NEXAFS) (Stöhr, 1992) provides a high degree of chemical sensitivity and provides readily quantifiable chemical information. 4D spectro-microscopic analysis has been achieved for environmental and biological (Obst et al. 2009, Obst \& Schmid 2014, Schmid et al. 2014) and polymer samples (Hitchcock et al. 2008). Although radiation damage rates vary greatly among different samples, for any given sample, when compared to electron energy loss spectroscopy in a transmission electron microscope (TEM-EELS), STXM provides lower radiation damage for a given core level spectroscopy measurement (Wang et al, 2009a,b). Thus STXM can offer advantages over analytical TEM for studies of radiation sensitive materials such as biological samples and polymers.

Currently, common algorithms used to reconstruct tomographic data sets include weighted back-projection (WBP) (Vainshtein 1970, Xu \& Wang 2005) and various iterative reconstruction methods, such as the simultaneous iterative reconstruction technique (SIRT) (Gilbert 1972, Penczek 2010). Both methods have been used previously in STXM tomography (Berejnov 2013). However, two key challenges arise in conventional STXM tomography when there is under-sampling in projection data acquisition. First, images are acquired at a limited number of discrete sample rotation angles (tilt angles) and so the information recorded is 
incomplete. The tilt increment and number of tilt angles is typically set by considerations of instrument and sample stability, radiation dose, and acquisition time. Second, samples for soft Xray STXM tomography must be relatively thin (less than $300 \mathrm{~nm}$ ) to allow X-ray penetration. When a flat sample is rotated to a high angle, the incident X-ray may be fully absorbed by the sample or may become shadowed by a support grid. As a result, $180^{\circ}$ rotation cannot be achieved, leaving a missing wedge of information in the tilt series. Typically, SIRT is more robust than WBP to the effects of the missing wedge and limited angular sampling (Gilbert 1972, Penczek 2010) and generally yields higher quality 3D reconstructions (Bangliang 2000).

Nevertheless, the highly under-sampled measurements typical of STXM tomography still lead to significant artefacts in both WBP and SIRT reconstructions.

The relatively recently-developed information recovery method known as compressed sensing (CS) can substantially reduce the number of measurements needed to recover a signal (Candès et al. 2006, Donoho 20006, Blumensath \& Davies 2009). CS and related methods have been deployed efficaciously to reduce the number of images needed for tomographic reconstruction (Sidky 2008), and to reduce artefacts arising from the missing wedge and thus reduce the overall dose needed for a given quality of 3D chemical imaging. CS has been shown to give high fidelity reconstructions even when very few tilt angles are used (Saghi et al., 2011, Leary et al., 2013, Saghi et al., 2016), as small as 9 angles in some cases (Saghi et al., 2011). In CS, prior knowledge of sample properties, such as the local homogeneity of constituent phases, is used to enable robust reconstructions from highly under-sampled measurements. CS approaches to electron tomography have demonstrated substantial achievements, including successful reconstructions of morphological and chemical signals from highly limited tilt series data (Haberfehlner et al. 2014, Nicoletti et al. 2013, Torruella et al. 2016). Here, we present CS tomography applied to 4D STXM analysis of highly radiation sensitive samples.

Many polymer samples are too beam sensitive for electron tomographic characterization. For these systems, chemical mapping is preferably performed in STXM. Proton exchange membrane fuel cells (PEM-FCs) represent a major emerging area in STXM characterization, and are industrially of considerable importance. PEM-FC technology is a promising green resource for stationary and automotive power applications (Folkesson et al. 2003, Wang et al. 2005). The oxygen reduction reaction at the cathode, in which oxygen, protons, and electrons combine to form water in a catalysed reaction, is the key rate-limiting step in PEM-FC. The cathode consists 
of carbon support particles decorated with Pt catalyst particles, surrounded by proton conducting perfluorosulfonic acid (PFSA) polymer (referred to as the ionomer when in the cathode) and inter-particle pores. Since a proton conductor such as PFSA is required to transport protons from the membrane-cathode interface throughout the cathode, the distribution of ionomer in the cathode plays a critical role in PEM-FC. Optimization of ionomer loadings and distributions requires direct observations of the internal structures and chemical states of the cathode catalyst layer in membrane electrode assemblies (MEAs). STXM has been applied as an effective method for mapping ionomer in 2D (Susac et al. 2011, Susac et al. 2013) and there has been some exploration of the potential for 3D ionomer mapping (Berejnov et al. 2013). The radiation sensitivity of PFSA has made it difficult to measure the 3D chemical structure, morphology, and quantitative distribution of ionomer in PEM-FC cathodes. Methods to reduce the dose by reducing the number of tilt angles are required for meaningful 3D chemical imaging.

In this work, we compare the results of WBP, SIRT, and CS reconstructions applied to several tomography data sets. In order to identify the benefits and characteristics of the compressed sensing approach, we have used readily available conventional approaches for the comparative WBP and SIRT analyses. First, we demonstrate the efficacy of CS tomography in the case of a high angle annular dark field scanning TEM (HAADF-STEM) tomography study of Au nanoparticles. This study highlights the performance of CS tomography in tilt series data comprising few projections and exhibiting the characteristic missing wedge in electron tomography and shows robustness to data corruptions including diffraction effects in crystalline samples. Second, a bilayer thin film was fabricated specifically to test the capability of STXM tomography to derive the correct 3D distribution of chemical components important in the analysis of PEM-FC electrodes. The model system contains components with spectral signatures similar to those in a PEM-FC cathode. A third sample, consisting of a complex polymer microsphere system (Johansson et al. 2007, Hitchcock et al. 2008) composed of a low density linear acrylate in a polystyrene shell inside a carbon nanopippette (Schrlau et al. 2008), illustrates the application of STXM tomography to a sample with multiple overlapping chemical spectral signatures present. Full $180^{\circ}$ rotation was possible for this sample, enabling an exploration of the effects of the size of the missing wedge on CS and other reconstruction methods. Together, these analyses establish CS tomography as a powerful technique for STXM tomography of radiationsensitive materials. 


\section{Methods}

\subsection{Electron Tomography}

Electron tomography tilt series data was acquired using an FEI Tecnai F20 FEG-TEM operated at $200 \mathrm{kV}$. Gold nanoparticles were prepared by a modified seed-mediated growth process as reported by Bandhopadhyay et al. (2017). The sample was drop cast onto 200 mesh copper TEM grids coated with a continuous carbon film. A tilt series of HAADF STEM images was acquired from $-68^{\circ}$ to $+64^{\circ}$ in $2^{\circ}$ increments. The tilt series image stack was first aligned using cross-correlation methods followed by alignment of the tilt axis, performed using Inspect3D (FEI).

\subsection{STXM tomography}

STXM tomography was carried out at the ambient STXM on beamline 10ID1 (SM) at the Canadian Light Source (CLS) and at beamline 5.3.2.2 at the Advanced Light Source (ALS). The tomography sample holder and rotation system was initially developed by Johansson et al. (2006). For the PEM-FC study in this work, we used an improved device with a smaller stepper motor and a simpler, more reliable mounting system (Schmid et al. 2014). In this system, the rotation is driven by a two-phase micro-stepper motor (Faulhaber ADM0620, Faulhaber, Schoenaich, Germany). The sample is a single strip cut from a 100 mesh, 3 mm TEM grid using a fresh scalpel. This strip is glued using nail polish to the tip of a $0.6 \mathrm{~mm}$ diameter brass rod, and the rod is mounted to the stepper motor using the chuck from a mechanical pencil using $0.6 \mathrm{~mm}$ graphite. The full sample width is around $250 \mu \mathrm{m}$. This narrow sample is necessary due to spatial constraints imposed by the order sorting aperture (OSA), which is only $250 \mu \mathrm{m}$ upstream from the X-ray focal point when using C 1s photon energies $(270-330 \mathrm{eV})$. The projection images were measured with the samples at room temperature, after the STXM tank was pumped to a residual vacuum of $\sim 1$ mbar, then back filled with He to 0.2 bar. Further details on the experimental methodology are included in table S-1 in the supplemental material.

The PEM-FC model system comprised a two-layer polymer film with Pt decorated carbon fibers in one layer and Teflon ${ }^{\mathrm{TM}}$ fibers in a second layer. The fibers in each layer were embedded in an epoxy. A STXM spectro-tomography data set was recorded from $-72^{\circ}$ to $+72^{\circ}$ at $4^{\circ}$ intervals. At each angle images were measured at four energies: 278 and $285 \mathrm{eV}$ at the $\mathrm{C} 1 \mathrm{~s}$ edge, to map the carbon fibers, and 684 and $694 \mathrm{eV}$ at the $\mathrm{F} 1 \mathrm{~s}$ edge, to map the Teflon ${ }^{\mathrm{TM}}$ fibers. 
The F 1s images were recorded before the $\mathrm{C} 1 \mathrm{~s}$ images to reduce the impact of radiation damage on the quantitation of the Teflon ${ }^{\mathrm{TM}}$.

The polymer microsphere sample (Johansson et al. 2007, Hitchcock et al. 2008) consisted of an aqueous suspension of $0.8 \mu \mathrm{m}$ diameter polymer particles enclosed in a carbon nanopipette (Schrlau et al. 2008). The wall of the carbon nanopipette consisted of fused multiwalled carbon nanotubes grown inside a tapered glass capillary. The tube wall was $45 \mathrm{~nm}$ thick and had an internal diameter which tapered from $3 \mu \mathrm{m}$ to $0.5 \mu \mathrm{m}$. The microspheres inside the nanopipette were composed of a rigid, hollow polystyrene (PS) shell which encapsulated a dilute linear polyacrylate (PA) water solution. The entire sample was initially suspended in water at the time of preparation. A significant portion of the water was lost during preparation and some of the acrylate diffused out of the microspheres, resulting in a layer of PA on the inner surface of the nanopipette. Images of the nanopipette sample were acquired for 23 energies from 282.5 to 306 $\mathrm{eV}$ in the $\mathrm{C} 1 \mathrm{~s}$ absorption edge. For tomography, the combined energy-image stack was acquired in a tilt series from $-90^{\circ}$ to $+90^{\circ}$ with a $4^{\circ}$ increment.

The image contrast in STXM is based on NEXAFS (Stöhr 1992), which provides sensitivity to the chemical structure due to creation of inner-shell excited and ionized states. In STXM, Xray absorption is measured by recording the transmitted signal $\left(\mathrm{I}_{\mathrm{t}}\right)$ as a function of photon energy. The transmitted signal is then converted to absorption signal (optical density, OD) by Lambert-Beer's law:

$$
\mathrm{OD}=\ln \left(\mathrm{I}_{0} / \mathrm{I}_{\mathrm{t}}\right)
$$

where $\mathrm{I}_{0}$ is the incident photon flux, typically measured in an area without the sample but with all underlying sample support materials present.

Axis2000 (Hitchcock 2016) was used for the initial analysis of both STXM data sets. After alignment and conversion from transmission to absorption, chemical maps at each angle were obtained from the difference of the peak energy image and the pre-edge energy image. Angle stacks consisting of chemical maps generated at every angle for each component were then assembled and further aligned. The full number of angles and parameters used in the SIRT and CS reconstructions are summarized in Table 1. 3D reconstruction software IMOD (Kremer et al. 1996) was used for WBP and SIRT reconstructions. A python-based implementation was used for the CS reconstructions. The CS code used in this study uses a total variation (TV) based, compressed sensing algorithm (https://github.com/emmanuelle/tomo-tv), employing a real space 
projection operator. It has been implemented in a dedicated X-ray spectromicroscopy processing tool called Mantis (Lerotic et al. 2014), available at http://spectromicroscopy.com. Mantis is an open source tool developed in Python for spectromicroscopy data analysis. Recently a new tab for spectro-tomography has been added which allows tomographic reconstruction of single energy datasets as well as energy-by-energy or component-by-component reconstruction. An image of the layout of the spectro-tomography interface in Mantis is provided in Figure S-1 of the supplemental material; both CS and SIRT are supported. The reconstructed 3D volume is visualized within Mantis by $x y, x z$ and $y z$ orthoslices. For spectro-tomography data, reconstructions can be done for each energy. In those cases spectral information for each voxel of the resulting volume is available and can be inspected with an ROI tool.

\subsection{Visualization}

Chimera (University of California, San Francisco, UCSF) (Pettersen et al. 2004) and Fiji (ImageJ) (Schneider et al. 2012), were used for volume rendering and visualization. The $z-$ direction labelled on the reconstruction visualisations is parallel to the optic axis (the missing wedge direction), the $y$-direction is parallel to the tilt axis and the $x$-direction perpendicular to these.

\subsection{Compressed sensing}

The concept of compression is pervasive in contemporary data handling, forming the foundation for important image file formats such as the ubiquitous JPEG format. The success of compression relies on the fact that most natural images can be described very well by a small number of coefficients in some basis. A basis consists of a set of functions that can be used to represent the image when combined with appropriate weighting factors or coefficients. Popular bases used for image compression are the discrete cosine transform (used in the JPEG format) and the discrete wavelet transform (used in the JPEG-2000 format). In the context of image compression, an image is first fully sampled (i.e., a measurement is recorded at each pixel in the image) before a transform is applied to the image data to determine the coefficients in the selected basis. To compress the image data, only the largest coefficients are retained and the small coefficients are discarded. Given that the majority of information in the image is contained in far fewer coefficients in the chosen basis than pixels in the original image, the data is said to 
be compressed. The stored coefficients can in turn be used to recover the image data with minimal information loss by inverting the transform used in the compression process.

In symbolic notation, the transform can be written as an operator $\Psi$, which, when applied to an image $\mathbf{x}$, returns a list of coefficients, a vector $\mathbf{c}$ :

$$
\mathbf{c}=\Psi \mathbf{x}
$$

If the coefficients are mostly zero, the vector $\mathbf{c}$ is said to be sparse. The number of non-zero elements in can be counted, $k$, and then the vector is said to be $k$-sparse. In compression, sparsity is enforced by setting small coefficients to zero, giving an approximate but nearly complete representation of the original image data. In this case, the coefficient vector is only approximately sparse, but the compressed image still contains the most important information.

In CS, the goal is to directly target the non-zero coefficients in c rather than the fully sampled image. If it can be assumed that the number of coefficients in $\mathbf{c}$ is small for a complete or nearly complete representation of the fully sampled data, then taking measurements directed at determining this small number of coefficients allows for taking fewer measurements during data acquisition. However, the way these few measurements are taken is critical in CS to ensure recovery of the most important coefficients in c.

In general, the signal measured experimentally is of the form

$$
\mathbf{b}=\Phi \mathbf{x}
$$

where $\mathbf{b}$ are the measurements recorded on the object $\mathbf{x}$. The operator $\Phi$ defines how the measurements are taken. In the case of tomography, $\Phi$ describes line integrals through the 3D object $\mathbf{x}$ which return projection data $\mathbf{b}$. In the framework of CS, the sampling equation (3) is modified to give

$$
\mathbf{b}=\Phi \Psi^{*} \mathbf{c}
$$

where $\Psi^{*}$ denotes the inverse transform. Equation (4) follows from combining equation (2) and equation (3).

Two key requirements underpin the mathematical theory for CS: (i) $\mathbf{x}$ is sparse in the transform domain $\Psi$ and (ii) $\Phi$ and $\Psi$ are incoherent. The requirement for sparsity emerges in a straight-forward way from the concept of compression. In order for a limited number of measurements to recover the important coefficients, it is critical that the number of coefficients sought is small. The concept of incoherence can be considered in two ways. In the first, incoherence ensures that individual measurements of $\mathbf{b}$ provide information about many 
coefficients of $\mathbf{x}$. In the second, the incoherence requirement can be understood to establish that artefacts due to under-sampling appear as noise during the reconstruction of $\mathbf{x}$, allowing the significant coefficients to stand out.

Given a set of measurements, CS aims to recover the sparse coefficient vector c. Direct minimization of the number of non-zero coefficients is generally computationally intractable. However, it has been shown that the computationally feasible problem of minimizing the $\ell_{1}$ norm returns the sparsest solution. The $\ell_{1}$-norm is defined as the sum of the absolute values of the $i$ elements of the vector $\mathbf{c}$ :

$$
\|\mathrm{c}\|_{\mathrm{i}}=\sum_{\mathrm{i}}\left|c_{\mathrm{i}}\right|
$$

The CS reconstruction can be written as a regularized minimization problem:

$$
\operatorname{argmin}_{\mathbf{x}}(\mathbf{x})=\left\{\|\Phi \mathbf{x}-\mathbf{b}\|_{2}^{2}+\beta\|\Psi \mathbf{x}\|_{1}\right\}
$$

where the squared $\ell_{2}$-norm, taken as

$$
\|\left.\mathrm{q}\right|^{2}=\sum_{\mathrm{i}} q_{\mathrm{i}}^{2}
$$

for any vector $\mathbf{q}$, compares the reconstructed signal $\mathbf{x}$ with the experimental measurements $\mathbf{b}$, and the second term, using the $\ell_{1}$-norm, promotes sparsity of the signal in a selected transform domain. In the combined minimization, the first term establishes data fidelity and the second term acts as a penalty term directing the reconstruction toward the sparsest solution. The factor $\beta$ is an adjustable weighting parameter that is tuned according to the intrinsic sparsity and the relative magnitudes of the signals in the minimization. In its most pure form, the CS approach is designed to overcome artefacts due to under-sampling.

The operator for the sparse transform domain, $\Psi$, should generally be selected in accordance with prior knowledge of the structure in the object. In the simplest case, if the object can be represented by a small, finite number of volume elements (voxels), then the identity transform would be a suitable operator, $\Psi$. Another sparse transform domain for many materials samples in the physical sciences is the gradient domain, since many samples consist of homogeneous regions of near constant density with sharp boundaries. In the gradient domain, such samples are represented by a small number of pixels at the boundaries between different homogeneous regions. Figure 1(a) illustrates such a transformation of an image into the gradient domain. The $\ell_{1}$-norm of spatial finite differences in an image serves to describe this sparse 
domain for computational purposes and is also called the total variation norm (TV-norm). For chemical samples, this TV-norm is applied in the spatial domain (typically for separated chemical phases); that is, the sparsity in the spatial gradient domain is used to improve tomographic reconstruction. Alternative sparsifying transforms may also be suitable for CS approaches in tomography, such as total generalized variation (Bredies et al. 2010), wavelets and related transforms (Mallat 2008), and adaptive strategies such as dictionary-learning techniques (Duarte-Carvajalino \& Sapiro 2009). In this study the sparseness of the data was described by the TV-norm that was applied with different degrees using the factor $\beta$ that was tuned for each dataset.

Regarding the second requirement for CS, that of incoherence, insight can be gained most readily by considering tilt series tomographic sampling in terms of a radial sampling of Fourier space via the Fourier slice theorem (Kak \& Slaney 1988), which seminal studies have shown satisfies the incoherence requirement (Lustig et al. 2007, Candes et al. 2006). The use of radial Fourier sampling in CS is further supported by recent theoretical work on optimized CS sampling regimes (Adcock et al. 2013). In this regard, CS tomographic reconstruction can be approached using Fourier or real space based projection operators, with the latter being used in this work. Figure 1 visually summarizes the algorithmic approach to solving equation (6) for the CS-TV tomography in this study, using a simple 2D phantom object similar to the PEM-FC material studied by STXM tomography.

\section{Results}

\subsection{Compressed Sensing Electron Tomography}

CS tomography has seen widespread application in electron microscopy to achieve high quality tomographic reconstructions from limited and challenging data sets. Here, in developing CS-TV methods for STXM tomography, we first highlight the performance of the reconstruction algorithm for handling missing wedge artefacts and diffraction contrast in electron tomography using a crystalline Au nanoparticle sample. This sample also provides a means for illustrating the robust performance of the CS-TV tomography approach when under-sampling a conventional tilt series by increasing the increment between tilt angles. Figure 2 compares orthogonal slices ('orthoslices') extracted from tomographic reconstructions using a common WBP and SIRT implementation (IMOD) with those from the CS-TV algorithm (Mantis). Fig. 2(a,b,c) shows the 
$x y$ slices for WBP, SIRT and CS-TV reconstructions from the full 67-angle set, while Fig. 2(d,e,f) presents the $x z$ slices of the same reconstruction. The $x z$ slices are emphasized as these show most prominently the artefacts resulting from the missing wedge due to the limited tilt range. Aside from angle-specific diffraction in $2 \mathrm{~d}$ images at specific tilt angles, there is good agreement between the $2 \mathrm{~d}$ projection generated from the reconstructions with the original data, as shown for a tilt angle of $0^{\circ}$ in Supplemental Figure S-2.

To compare the reconstruction results with a reduced number of tilt-angles, sub-sets of 23 angles (with $6^{\circ}$ increments) and 12 angles (with $12^{\circ}$ increments) were analyzed. As summarized in Fig. 2(d-i), the WBP and SIRT reconstructions for all three sets of projection angles used showed 'streaking' artefacts, characteristic of the under-sampling between adjacent tilts and the missing wedge. The surface of the Au particles became less well defined as the number of tilt angles was decreased. In contrast, the results from the CS-TV reconstruction were similar, and overall of much higher quality, for all three sampling regimes. The $x y$ slices for both SIRT and CS-TV reconstructions showed some banding in the intensities throughout the volume, which are attributed to diffraction contrast effects, i.e. modulations of signal intensity at or near Bragg conditions of the Au crystals. While for an individual particle such diffraction contrast contributions would only modulate the relative intensity of the particle, in the ensemble of particles which overlap significantly at high tilt the effect of the diffraction contrast gives rise to the observed bands. The difference in brightness of particles should not be interpreted as missing intensity but rather is a result of inconsistent intensities in the input tilt-series.

The CS-TV reconstruction deals with the diffraction artifacts in the tilt-series somewhat differently from the WBP and SIRT reconstructions in that the CS-TV reconstructions show larger regions of flatter intensity. At the top and bottom of the orthoslice in Fig. 2(c), particles appear relatively brighter than in the middle of the particle aggregate, due to changes in the total signal magnitude relative to the TV-regularization parameter for each two-dimensional reconstruction in the planes perpendicular to the tilt axis (see also Sec. 2.4). Three-dimensional TV regularization methods, additionally incorporating the gradient along the tilt axis in the TVnorm, have been applied in electron tomography of samples exhibiting high levels of diffraction contrast, and these three-dimensional CS-TV methods further reduce diffraction effects by promoting particle densities that are homogeneous throughout the full reconstruction volume (Leary et al. 2016). However, there is a trade-off in the computational efficiency in 3D 
compared to $2 \mathrm{D}$ reconstruction which may be undesirable for applications in spectroscopic STXM tomography where computational requirements are already higher due to the use of signals at multiple energies.

Figure 3 presents line intensity profiles in horizontal and vertical directions from the reconstructions for all three tilt angle ranges. The WBP vertical profile shows a high noisy background along the vertical missing wedge direction, in agreement with Fig. 2(d,g,j). For the SIRT reconstructions, the intensity in the horizontal profile is reduced as the number of projections is reduced. However there is no significant change in the CS profiles as the number of projections is reduced. Both the horizontal and vertical profiles of CS have very low background intensity and the surfaces of the Au particles are well reconstructed. We note that the SIRT analysis can be improved. There are many different ways to implement SIRT. Alternative SIRT strategies include those described in the overview by Elfving et al, (2012), which outlines the approaches first reported by Cimmino 1938, and Landweber et al. 1951. Other iterative based methods such as those proposed by Anderson and Kak, 1984, Gregor and Benson, 2008, and Censor et al 2008, may offer further improvements to a SIRT type of analysis. Any one of these may work better or worse for a given data-set. We are using a readily available and widely used SIRT implementation. Our main goal was to show the difference between a typical SIRT implementation and what the addition of prior knowledge in the form of TV in CS does in terms of the reconstruction quality. Supplemental Figures S-3 and S-4 present SIRT results with addition of compact support and non-negativity constraints. When these same approaches are also applied to the CS analysis, there is still clearly a significant benefit to using CS. Adding the non-negativity constraints improves the SIRT results. In the same way there are also be other ways of improving CS, including applying non-negativity. It is critical that fair comparisons be made and these comparisons should include equivalent constraints (only change one thing at a time, in this case moving from SIRT to CS is meant to only add the TV constraint). In fact, CS continues to develop as a field, suggesting other possible improvements to come that are beyond the scope of the present work.

These electron tomography results illustrate key performance characteristics of CS reconstruction methods. They present a useful starting point for transferring CS tomography approaches to STXM and for comparing performance characteristics in considering missing wedge and under-sampling artefacts common to electron and X-ray tomography. 


\subsection{STXM tomography of a model PEM-FC sample}

Figures $\mathbf{4} \mathbf{a}$ and $\mathbf{4 b}$ shows the individual component $\mathrm{X}$-ray absorption reference spectra used to analyse the bilayer model carbon-fluorine fiber sample. For spectral analysis, and to explore an optimized 2D projection analysis for comparison to 3D chemical imaging using a restricted set of photon energies, a region of the bilayer sample was measured over a range of photon energies at the $\mathrm{C} 1 \mathrm{~s}$ and $\mathrm{F} 1 \mathrm{~s}$ edges. Data was acquired in the form of a sequence of images at varying photon energies, also called stacks (Jacobsen et al. 2000) on a transverse cross-section of the bilayer sample. After alignment, the intensity at a given pixel through the stack gives a transmission spectrum of the material at that pixel. The C-fiber map (Fig. 4c) was generated from the $\mathrm{C}$ 1s stack data while the F-fiber (Teflon ${ }^{\mathrm{TM}}$ ) map (Fig. 4e) was generated from the F 1s stack data, in each case using stack fit (Hitchcock 2012), a quantitative chemical analysis process in which reference spectra and a constant are used in a singular value decomposition (Zhang et al. 1996) of the multi-energy absorption data set. The Pt-shell map (Fig 4d) was derived from the constant map of the $\mathrm{C} 1 \mathrm{~s}$ stack fit. Fig. $\mathbf{4 f}$ presents a color coded composite highlighting the distribution of Teflon ${ }^{\mathrm{TM}}$ relative to the distributions of the carbon fiber and the Pt shell. Signals arising from the carbon fiber are shown in red, the Teflon ${ }^{\mathrm{TM}}$ fiber is shown in green, and the Pt shell is shown in blue. Areas without color correspond to regions of embedding resin. From the full $\mathrm{C} 1 \mathrm{~s}$ and $\mathrm{F}$ 1s spectra, component-specific peak energies and preedge energies were identified. The first peak in the $\mathrm{C} 1 \mathrm{~s}$ spectrum corresponds to $\mathrm{C} 1 \mathrm{~s} \rightarrow \pi^{*}$ transitions, which only occur in the graphitic carbon fiber. Thus the carbon fiber map was obtained by subtracting a pre-edge absorption image $(278 \mathrm{eV})$ from the image at the $\pi^{*}$ peak $(285.3 \mathrm{eV})$. The Teflon ${ }^{\mathrm{TM}}$ fibers, simulating the perfluorosulfonic acid (PFSA) component of PEM-FC electrodes, were selectively probed at the F 1s edge, since the Teflon ${ }^{\mathrm{TM}}$ fibers are the only component containing fluorine. The $\mathrm{C} 1 \mathrm{~s}$ pre-edge image $(278 \mathrm{eV})$ was dominated by contributions from Pt and F. An estimate for the Pt signal was generated from the $\mathrm{C}$ 1s pre-edge image and the F 1s map using the procedure outlined by Hitchcock et al. (2014). In order to limit radiation damage, tilt series images were recorded only at 278.0,285.3, 684.0 and $693.0 \mathrm{eV}$ at each scan angle. Due to limited penetration at high angles, the maximum rotation angle was $\pm 72^{\circ}$, and this range was sampled with a $4^{\circ}$ increment.

In order to compare the reconstruction quality of WBP, SIRT, and CS-TV algorithms with varied sub-sampling, two subsets from the complete 37 projection set, consisting of 19 
projections and 10 projections, were reconstructed by each method. Figure 5 presents $x y$ and $x z$ orthoslices from the 3D reconstructed volume for the carbon fiber. The other components showed similar results and are displayed as multi-component surface renderings in Figure 6. When all 37 projection tilt angles were used, the WBP and SIRT reconstructions reproduced the carbon support fiber distribution to a reasonable approximation (Fig. 5a,d). However, even at this degree of sampling, the CS-TV reconstructions were of substantially higher quality, with a much lower level of artefacts than the SIRT result, greatly easing interpretation of the carbon fiber support morphology (Fig. 5g). When profiles along the A-B line in xy and the C-D line in Xz slices were compared, the intensity distribution from the WBP reconstruction was much lower than that in the SIRT and CS reconstructions, and the SIRT reconstruction contained more negative signal. As the tilt increment was increased and the number of projections decreased, the quality of the reconstruction results from the WBP and SIRT methods showed substantial degradation. This was especially noticeable in the $x z$ slices (Fig. 5 c,f lower) revealing missing wedge effects. Streaking artefacts were more prominent, blurring in the missing wedge direction was greater, the particles became elongated in the missing wedge direction, and gray-levels were more distributed throughout the volume. In the $x y$ slices (Fig. 5b,e,c,f upper), both the density and the shape were distorted. In contrast to the WBP and SIRT results, the CS-TV reconstructions did not show substantial changes using 19 (Fig. 5h) or only 10 projections (Fig. 5i). For the CS-TV reconstructions, the distribution and morphology of the carbon fibers remained nearly identical to that in the reconstruction using the full projection series. Similar conclusions can be obtained from the line profile plot results. As the number of projections was decreased, the WBP and SIRT reconstruction line profiles changed, especially for the 10projection reconstruction, while the line profiles for the 10-projection CS reconstruction were similar to those from the 37-projection reconstruction. The intensity of the SIRT reconstruction signal decreased by $\sim 50 \%$ from the 37 - to the 10 -projection, and the background became noisier. The success of CS-TV reconstruction relative to WBP and SIRT became increasingly pronounced as the number of projections was reduced.

Figure 6 presents color-composite isosurface renderings of WBP, SIRT, and CS-TV reconstructions for all three chemical components. Note that the epoxy contributed minimally to the Teflon ${ }^{\mathrm{TM}}$ fiber, carbon fiber or Pt maps because those maps were derived from optical density differences at energies where the spectrum of the epoxy does not change (Fig. 4a). In Figs. 4 and 
6, the empty regions are uniformly filled with epoxy. The isosurface renderings were obtained using the Otsu automatic threshold method (Otsu 1975) implemented in ImageJ. Fig. 6 compares $0^{\circ}$ and $90^{\circ}$ views of the CS-TV and SIRT reconstructions shown in Fig. 5, and reinforces the trends seen there. For the full 37 tilt angle set (Fig. 6a,d), the SIRT, CS-TV and WBP reconstructions exhibited similar morphology and spatial distributions for the three components. However, even with the full set of angles, the WBP reconstruction showed high background levels (Fig. 6g). The isosurfaces showed compact structures with sharp edges, consistent with a relatively high signal-to-noise ratio (SNR) in the reconstruction volume. The results from CS-TV for the 19-tilt angle (Fig. 6b) and 10-tilt angle (Fig. 6c) sets remained of high quality, showing only small changes relative to the analysis of the 37-tilt angle set. However, in the corresponding SIRT reconstructions, substantial speckle and a gradual smearing of the edges appeared in the reconstructions as the number of projections was reduced (Fig. 6e-f). The signal from the WBP reconstructions of 19- projection (Fig. 6h) and 10-projection (Fig. 6i) sets was basically lost in the noise. The missing wedge problem strongly affects WBP, as has been noted elsewhere (Penczek 2010).

As highlighted in equation (6), the CS-TV method requires the selection of the parameter $\beta$, the weighting factor for the TV-norm in the reconstruction process. In principle, the optimal value for $\beta$ is defined by the intrinsic sparsity of the object and the relative magnitudes of the signals in the reconstruction. Experimentally, the value of $\beta$ will also be influenced by noise or other artefacts in the data set. Moreover, the true sparsity of the object will not be known in general. As a result, $\beta$ must be optimized for each data set. Figure 7 illustrates the effects of different choices of $\beta$ for CS-TV reconstruction of the 37-projection data set of the bilayer model sample (carbon fiber map). When $\beta$ was set too low (Fig. 7a,d) there was little effect of TV regularization and the reconstruction showed negligible benefit of CS. When $\beta$ was set too high (Fig. 7c,f), the effect of the TV penalty term was over-emphasized, resulting in a flattened image with plateaus in the reconstructed signal and background which did not represent physical structures in the sample. This is clearly seen in the line profiles across the projections (Figs. 7g,h).

In general, the optimal $\beta$ value will be larger for greater signal magnitudes, e.g., if more projections are included in the input data set. In this case, the data fidelity term in equation (6) is larger, and the penalty term requires greater weight to have the same effect. The sparsity of the 
object will also affect the optimal $\beta$, as will the noise level and the presence of other inconsistencies in the data. Although these heuristics provide some guidelines for selecting $\beta$, often empirical optimization of the $\beta$ parameter is needed. In principle, the $\beta$ parameter could be normalized relative to the term accounting for consistency with the experimental data, removing some of the dependence on the number of tilt-angles. The implementation presented here maintains the behaviour of the $\beta$ parameter from earlier work for consistency in the application of CS-TV to STXM tomography. For a day-to-day user, the effect remains the same: $\beta$ is necessarily optimized for the particular data-set at hand. Typically, this optimization may be carried out on a representative $x z$ slice from a given sample, and then the more time- and computationally-intensive volume reconstruction can be carried out with the selected optimal $\beta$ value.

\subsection{D mapping acrylate/polystyrene in a nanopipette}

The microsphere-nanopipette sample allowed exploration of CS-TV tomography using a data set with a richer spectral dimension than that of the bilayer model sample. This case exhibits gradient domain sparsity (TV-sparsity) in the spatial dimensions, making use of a separate decomposition of the finite number of chemical phases in the spectral dimension. In this sample, multiple carbon-based compounds with overlapping signals were present, demonstrating the power of 4d imaging using STXM spectro-tomography. Additionally, the needle-like sample geometry enabled evaluation of CS-TV tomography with a data set acquired over a complete $180^{\circ}$ tilt range, which allowed examination of the effect of varying the angular range by artificially introducing a missing wedge post facto.

The C 1s spectra of the components extracted from the C 1s stacks are shown in Fig. 8a. The X-ray absorption was saturated at several photon energies around the intense $285 \mathrm{eV} \mathrm{C} 1 \mathrm{~s} \rightarrow$ $\pi^{*}$ peak because both the carbon nanotube pipette and polystyrene (PS) absorb strongly at this energy, so that the total absorption became too large when the X-rays passed through more than one microsphere. Figure 8b-d presents the chemical maps for the acrylate, PS, and carbon nanopipette components derived from SVD analysis of one angle projection, using all energies except those at 285.0 and $285.5 \mathrm{eV}$ where the absorption saturation distortion occurs. Fig. 8e is a color composite of these three component maps. The carbon nanopipette map was expected to show signal from the exterior tube only. The acrylate should show a larger density inside the 
microspheres, although it is known that acrylate leaked from some of the hollow PS particles in this particular sample (Johansson et al, 2007). If so, as the water was lost, the acrylate dried on to the interior of the PS shell particles or onto the interior of the carbon nanopipette.

Figure 9 presents the 3D reconstructions of the acrylate maps obtained using WBP, CSTV and SIRT for tilt angle ranges of $\pm 90^{\circ}, \pm 74^{\circ}$, and $\pm 62^{\circ}$. The results from the $\pm 90^{\circ}$ tilt set showed hollow acrylate distributions inside the tube in the $x y$ slice and no missing wedge artefacts in the $x z$ slice (Fig 9a,d,g). Using presently available STXM tilt-series data-acquisition technology and standard processing techniques (Hitchcock 2016), WBP and SIRT methods yielded reconstructions with intensities with both negative and positive values. Such artefacts represent a key limitation for analytical tomography where intensity represents concentration, motivating the development of alternative reconstruction techniques. In contrast, using only the addition of the TV-minimization constraint in CS, the reconstructions were significantly improved, with physically interpretable intensities. When the analysis was performed using reduced tilt angle ranges, missing wedge artefacts appeared in the WBP, SIRT and CS-TV reconstructions, though the artefacts were much less severe in the CS-TV reconstructions (Fig. 9g,h,i). Figure 10 presents line intensity profiles along the missing wedge direction for the WBP (Fig. 10a), SIRT (Fig. 10b) and CS-TV (Fig. 10c) results for each of the three tilt angle ranges. For WBP and SIRT reconstructions, the line profiles showed significant broadening in the missing wedge direction as the size of the missing wedge was increased (highlighted with arrows in Fig. 10a,b). The intensity distributions in line profiles from the $\pm 90^{\circ}$ and $\pm 74^{\circ}$ tilt sets reconstructed using CS-TV were similar (Fig. 10c). For CS-TV reconstructions, effects of the missing wedge appeared as a slight broadening of features only when the tilt angle range was reduced to $\pm 62^{\circ}$. Moreover, changes appeared in isolated regions of the CS-TV reconstructions, whereas for SIRT reconstructions all peaks were affected by the increased missing wedge. Overall, CS-TV reconstructions maintained data fidelity to a reasonable level, whereas WBP and SIRT showed a significant reduction in reconstruction quality. Supplemental Figure S-5 presents WBP, SIRT and CS reconstructions of the images recorded at a single photon energy $(290 \mathrm{eV})$, in order to explore if the analysis in the spectral domain to generate component maps had an effect. When only a single energy is analysed, the clarity of the reconstructions is improved for each method, but similar results with respect to the relative quality of WBP, SIRT and CS are obtained as for the reconstructions of the component maps. Supplemental Figure S- 
6 presents SIRT analyses with a non-negativity constraint of the polystyrene and carbon nanotubes component maps, at threes different tilt angle ranges $\left( \pm 90^{\circ}, \pm 74^{\circ} \pm 62^{\circ}\right)$. As found for the Au dumbbell electron tomography analysis, applying the non-negativity constraint does improve the SIRT reconstructions and reduces its sensitivity to changes in the tilt angle range analysed.

\section{Discussion}

The demonstration of a CS-TV tomography algorithm for STXM presented here outlines ways for improving the quality of STXM tomography reconstructions and opens up new possibilities for dose-rationing in STXM tilt series spectro-tomography. The geometry of STXM optics means that many samples impose an upper limit on the tilt-range accessible for tomography, introducing a missing wedge of information, as in electron tomography. The CSTV approach applied to STXM tilt series data of a PEM-FC model sample and a chemically complex microsphere sample has shown significant benefits over conventional WBP and SIRT methods for data sets with a missing wedge.

Furthermore, the CS-TV tomography approach has been shown to be robust to substantial under-sampling by increasing the angular increment in a tilt series. For STXM of radiationsensitive materials, this CS-TV approach makes available several new acquisition strategies for minimizing dose. For a given SNR in each tilt series projection, the total dose may be reduced by acquiring fewer images at larger tilt increment. Alternatively, for a fixed total dose, the SNR of each individual projection may be increased by acquiring fewer images, each for a longer time. Each of these approaches may offer advantages for different samples, for imaging at different ionization edges, or at different spatial resolutions. Critically, enabling these techniques will allow 3D chemical imaging of samples that cannot be imaged with electron tomography, even with CS techniques, due to their radiation sensitivity. Our model sample analyses demonstrate the capability of CS-TV to produce high fidelity tomographic reconstructions using a very small number of tilt angles. The feasibility of STXM tomography using limited projection data is a critical and promising result for 3D chemical imaging of the ionomer in PEM-FC cathodes, since PFSA is highly radiation sensitive (Susac et al 2013, Melo et al. 2016) and excessive mass loss and ionomer redistribution can occur when more than $\sim 10$ angles are measured. We anticipate that CS-TV methods will become widespread and routine in STXM tomography, particularly for 
radiation sensitive materials such as the ionomer in PEM-FC cathodes. Reliable mapping of PEM-FC cathodes, and the corresponding quantitative reliability of 3D mapping of PFSA will be possible with 12-15 projections instead of 30-40 projections or more, as in more conventional approaches to STXM tomography.

At its core, the CS-TV algorithm is widely adaptable to many materials samples. The TVnorm requires the sample to be composed of at least approximately homogeneous chemical phases. This assumption is readily satisfied by many chemistries where individual compounds are present in separate sub-volumes. The CS-TV approach is, moreover, often more flexible than other advanced tomographic approaches like the discrete algebraic reconstruction technique (DART) (Batenburg and Sijbers 2007). DART assumes a known, finite number of grey levels in the reconstruction volume. The CS-TV approach does not impose knowledge of the number of different homogeneous phases. On the other hand, samples containing continuous chemical gradients, for example, may not be suitable for CS-TV STXM tomography methods. In those cases the CS framework allows alternatives to the TV-norm to be used for CS reconstructions. Identity and wavelet transforms have been applied to cases in electron tomography (Saghi et al, 2011, Nicoletti et al. 2013) and these sparsity transforms can be combined for improved results (Leary et al. 2012, Saghi et al. 2016). Total generalized variation techniques (Al-Aleef et al. 2015a) and dictionary learning approaches (Al-Aleef et al. 2015b, Al-Aleef et al. 2016) to CS tomography also present alternatives for dealing with continuous intensity variations and other types of structure in data. By applying transform domains that are appropriate to the structure in the data, CS can be widely adapted to different types of samples. Here, the CS-TV approach is broadly applicable to many common sample types encountered in STXM.

The high chemical sensitivity of spectroscopic STXM, well illustrated in the microsphere sample, represents another key advance for 3D chemical imaging. The wealth of chemical detail available in NEXAFS can now be recovered for 3D micro- and nano-scale analysis using advanced tomography techniques like the CS-TV algorithm. The many STXM modalities, including developments in ptychographic STXM imaging, will likely also benefit from combination with CS-TV tomography methods. 


\section{Summary}

An implementation of the CS-TV algorithm for analysis of STXM tomography data sets was presented, and its performance was evaluated relative to WBP and SIRT analyses of three different data sets. In each case, significant improvements were demonstrated, especially for highly under-sampled tilt series. The results show that CS-TV can be used to derive high fidelity 3D morphology even when radically fewer projections are used. This approach provides a method to improve the reliability of 3D mapping of ionomer in PEM-FC cathodes and many other radiation sensitive materials by using fewer projections and thus decreasing radiation damage, and it should also be applicable to ptychographic tomography (Holler et al 2014) and spectro-ptycho-tomography.

\section{Acknowledgements}

We thank Marcia Reid (McMaster University) who prepared samples and Martin Obst (University of Bayreuth) who made the tomography rotation stage. We are grateful for excellent instrumentation support from CLS staff, Jian Wang and Jay Dynes; and the ALS staff, Tolek Tyliszczak, and David Kilcoyne. Tomography and other data were acquired at beamlines 5.3.2.2 and 11.0.2 at the Advanced Light Source, which is supported by the Director of the Office of Science, Department of Energy, under Contract No. DE-AC02-05CH11231. Some results were also obtained with the ambient STXM on beamline 10ID1 at the Canadian Light Source, which is supported by the Canadian Foundation for Innovation. The research leading to these results has received funding from the European Research Council under the European Union's Seventh Framework Programme (FP7/2007-2013)/ERC grant agreement 291522-3DIMAGE, as well as from the European Union Seventh Framework Programme under Grant Agreement 312483ESTEEM2 (Integrated Infrastructure Initiative - I3). R.L. acknowledges a Junior Research Fellowship from Clare College.

\section{References}

ADCOCK, B.; HANSEN, A.C.; POON, C.; ROMAN, B. (2013) Breaking the coherence barrier: A new theory for compressed sensing. arXiv:1302.0561 
ADE, H.; HITCHCOCK, A.P. (2008) NEXAFS microscopy and resonant scattering:

Composition and orientation probed in real and reciprocal space. Polymer, 49, 643-675.

AL-AFEEF, A., ALEKSEEV, A., MACLAREN, I., AND COCKSHOTT, P. (2015) Electron tomography based on a Total Generalized Variation minimization reconstruction technique. 31st Picture Coding Symposium, Cairns, Australia.

AL-ALEEF, A.; COCKSHOTT, W.P.; MACLAREN, I.; MCVITIE, S. (2015b) Electron tomography image reconstruction using data-driven adaptive compressed sensing. Scanning 38, 251-276.

AL-ALEEF, A.; BOBYNKO, J.; COCKSHOTT, W.P.; CRAVEN, A.J.; ZUAZO, IU.; BARGES, P.; MACLAREN, I. (2016) Linear chemically sensitive electron tomography using DualEELS and dictionary-based compressed sensing. Ultramicroscopy 170, 96-106.

ANDERSEN, A.H.; A.C. KAK (1984) Simultaneous algebraic reconstruction technique (SART): A superior implementation of the ART algorithm, Ultrasonic Imaging, 6, 81-94.

BANDHOPADHYAY, S.; SINGH, G.; GLOMM, W. R. (2017) Shape tunable synthesis of anisotropic gold nanostructures through binary surfactant mixtures, Materials Today Chemistry, 3, 1-9.

BANGLIANG, S.; YIHENG, Z.; LIHUI, P.; DANYA, Y.; BAOFEN, Z. (2000) The use of simultaneous iterative reconstruction technique for electrical capacitance tomography. Chemical Engineering Journal 77, 37-41.

BARUCHEL, J.; BUFFIERE, J.-Y.; CLOETENS, P.; DI MICHIEL, M.; FERRIE, E.; LUDWIG, W.; MAIRE, E.; SALVO, L. (2006) Advances in synchrotron radiation microtomography. Scripta Materialia, 55, 41-46. 
BATENBURG, K.J.; SIJBERS, J. (2007) Dart: A Fast Heuristic Algebraic Reconstruction Algorithm for Discrete Tomography. 2007 IEEE International Conference on Image Processing, 10.1109/ICIP.2007.4379972.

BEREJNOV, V.; SUSAC, D.; STUMPER, J.; HITCHCOCK, A.P. (2013) 3D Chemical Mapping of PEM Fuel Cell Cathodes by Scanning Transmission Soft X-ray SpectroTomography. ECS Transactions 50, 361-368.

BLUMENSATH, T; DAVIES, M.E. (2009) Iterative hard thresholding for compressed sensing. Applied and Computational Harmonic Analysis 27, 265-274.

BOUDIN, F., HOURS, M. LACRONIQUE, J.-F. SALVO, L. SUÉRY, M. MARMOTTANT, A. LIMODIN, N. \& BERNARD, D. (2010) 3D imaging in material science: Application of X-ray tomography. Comptes Rendus Physique, 11, 641-649.

BREDIES, K.; KUNISH, K.; POCK, T. (2010) Total Generalized Variation. SIAM J. Imaging Sci. 3, 492-526.

CANDÈS, E.J.; ROMBER, J.; TAO, T. (2006) Robust Uncertainty Principles: Exact Signal Reconstruction From Highly Incomplete Frequency Information. IEEE Transactions on Information Theory 52, 489-509.

CENSOR, Y.; ELFVING, T.; HERMAN, G. T.; NIKAZAD, T. (2008) On diagonally relaxed orthogonal projection methods, SIAM J. Sci. Comput., 30, 473-504

G. CIMMINO, G. (1938) Calcolo approssimato per le soluzioni dei sistemi di equazioni lineari, La Ricerca Scienti ca, XVI, Series II, Anno IX, 1,326-333.

D. L. DONOHO, D.L. (2006) Compressed sensing, IEEE Transactions on Information Theory 52, 1289-1306. 
CHAO, W., FISCHER, P.; TYLISZCZAK, T.; REKAWA, S.; ANDERSON, E.; NAULLEAU, P. (2012) Real space soft x-ray imaging at $10 \mathrm{~nm}$ spatial resolution. Optics Express 20, 97779783.

DUARTE-CARVAJALINO, J.M.; SAPIRO, G. (2009) Learning to Sense Sparse Signals: Simultaneous Sensing Matrix and Sparsifying Dictionary Optimization. IEEE Transactions on Image Processing 18, 1395-1408.

ELFVING, T.; HANSEN, P. C.; NIKAZAD, T. (2012) Semiconvergence and relaxation parameters for projected SIRT algorithms, SIAM J. Sci. Comput., 34, A2000-A2017.

ERCIUS, P.; ALAIDI, O.; RAMES, M.J.; REN, G. (2015) Electron Tomography: A ThreeDimensional Analytic Tool for Hard and Soft Materials Research. Advanced Materials 27, 5638-5663.

FOLKESSON, A.; ANDERSSON, C.; ALVFORS, P.; ALAKÜLA, M.; OVERGAARD, L. (2003) Real life testing of a Hybrid PEM Fuel Cell Bus. J. Power Sources 118, 349-357.

GILBERT, P. (1972) Iterative methods for the three-dimensional reconstruction of an object from projections. J. Theoretical Biology, 36, 105-117.

GREGOR, J.; BENSON, T. (2008) Computational Analysis and Improvement of SIRT, IEEE Transactions on Medical Imaging, 27, 918-924.

HABERFEHLNER, G.; ORTHACKER, A.; ALBU, M.; LI, J.; KOTHLEITNER, G. (2014) Nanoscale voxel spectroscopy by simultaneous EELS and EDS tomography. Nanoscale $\mathbf{6}$, 14563-14569.

HITCHCOCK, A.P. (2012) Soft X-Ray Imaging and Spectromicroscopy, in Handbook of Nanoscopy. Wiley-VCH Verlag GmbH \& Co. KGaA. pp. 745-791. 
HITCHCOCK, A.P.; BEREJNOV, V.; LEE, V.; WEST, M.M.; DUTTA, M.; COLBOW, V.; WESSEL, S. (2014) Carbon corrosion of Proton Exchange Membrane Fuel Cell catalyst layers studied by Scanning Transmission X-ray Microscopy, J. Power Sources 266, 66-78.

HITCHCOCK, A.P. (2016) The program aXis2000 (Analysis of X-ray Images and Spectra) is written in IDL, and is available from http://unicorn.mcmaster.ca/aXis2000.html, free for noncommercial applications.

HITCHCOCK, A.P.; JOHANSSON, G.A.; MITCHELL, G.E.; KEEFE, M.H.; TYLISZCAK, T. (2008) 3-d chemical imaging using angle-scan nanotomography in a soft X-ray scanning transmission X-ray microscope. Applied Physics A 92, 447-452.

HOLLER, M.; DIAZ, A.; GUIZAR-SICAIROS, M.; KARVINEN, P.; FÄRM, E.; HÄRKÖNEN, E.; RITALA, M.; MENZEL, A.; RAABE, J.; BUNK, O. (2014) X-ray ptychographic computed tomography at $16 \mathrm{~nm}$ isotropic 3D resolution. Scientific Reports $\mathbf{4}$, 3857.

HOWELlS, M.; JACOBSEN, C.; WARWICK, T.; VAN DEN BOS, A. (2007) Principles and Applications of Zone Plate X-Ray Microscopes, in Science of Microscopy, P.W. Hawkes and J.C.H. Spence, Editors. Springer New York: New York, NY. pp. 835-926.

JACOBSEN, C.; WIRICK, S. ; FLYNN, G.; ZIMBA, C. (2000) Soft X-ray Spectroscopy from Image Sequences with Sub-100 nm Spatial Resolution. J. Microscopy 197, 173-184.

JOHANSSON, G.A.; DYNES, J.J.; HITCHCOCK, A.P.; TYLISZCZAK, T.; SWERHONE, G.D.; LAWRENCE, J.R (2006) Chemically Sensitive Tomography at $50 \mathrm{~nm}$ Spatial Resolution using a Soft X-ray Scanning Transmission X-Ray Microscope. Microscopy and Microanalysis 12, 1412-1413.

JOHANSSON, G.A.; TYLISZCZAK, T.; MITCHELL, G.E.; KEEFE, M.H.; HITCHCOCK, A.P. (2007) Three-dimensional chemical mapping by scanning transmission X-ray spectromicroscopy. J Synchrotron Rad. 14. 395-402. 
KAK, A.C.; SLANEY, M. (1988) Principles of computerized tomographic imaging, Society for Industrial and Applied Mathematics, (IEEE Press: New York).

KREMER, J.R.; MASTRONARDE, D.N.; MCLNTOSH, J.R. (1996) Computer visualization of three-dimensional image data using IMOD. J. Structural Biology 116, 71-76.

LANDWEBER, L. (1951) An iteration formula for Fredholm integral equations of the first kind, American Journal of Mathematics, 73, 615-624.

LEARY, R.; MIDGLEY, P.A.; THOMAS, J.M.(2012) Recent Advances in the Application of Electron Tomography to Materials Chemistry. Accounts of Chemical Research 45, 1782-1791.

LEARY, R., SAGHI , Z., MIDGLEY, P.A. \& HOLLAND, D.J. (2013) Compressed sensing electron tomography. Ultramicroscopy 131, 70-91.

LEARY, R.K.; KUMAR, A. STRANEY, P.J.; COLLINS, S.M.; YAZDI, S.; DUNINBORKOWSKI, R.E.; MIDGLEY, P.A.; MILLSTONE, J.E.; RINGE, E. (2016) Structural and Optical Properties of Discrete Dendritic Pt Nanoparticles on Colloidal Au Nanoprisms. J. Phys. Chem C 120, 20843-20851.

LEROTIC, M.; MAK, R.; WIRICK, S.; MEIRER, F.; JACOBSEN, C. (2014) MANTiS: a program for the analysis of X-ray spectromicroscopy data. J. Synchrotron Radiation 21, 12061212.

LUSTIG, M.; DONOHO, D.; PAULY, J.M. (2007) Sparse MRI: The application of compressed sensing for rapid MR imaging. Magnetic Resonance in Medicine, 58, 1182-1195.

MALLAT, S. (2008) A Wavelet Tour of Signal Processing. 3rd Ed. (Academic Press, . Cambridge, MA)

MELO, L.G. A.; HITCHCOCK, A.P.; BEREJNOV, V.; SUSAC, D.; STUMPER, J.; BOTTON, G.A. (2016) Evaluating focused ion beam and ultramicrotome sample preparation for analytical 
microscopies of the cathode layer of a polymer electrolyte membrane fuel cell. J.Power Sources 312, 23-35.

NICOLETTI O.; DE LA PEÑA F.; LEARY R.K.; HOLLAND D.J.; DUCATI, C.; MIDGLEY P.A. (2013) Three-dimensional imaging of localized surface plasmon resonances of metal nanoparticles. Nature, 502, 80-84.

OBST, M.; WANG, J.; HITCHCOCK, A.P. (2009) Soft X-ray spectro-tomography study of cyanobacterial biomineral nucleation. Geobiology, 7 577-591.

OBST M.; SCHMID G. (2014) 3D chemical mapping: application of scanning transmission (soft) X-ray microscopy (STXM) in combination with angle-scan tomography in bio-, geo-, and environmental sciences. in: Kuo J (ed) Electron microscopy. (Humana Press, New York) 757-781 OTSU, N. (1975) A threshold selection method from gray-level histograms. Automatica 11.285296, 23-27.

PENCZEK, P.C. (2010) Fundamentals of three-dimensional reconstruction from projections. Methods Enzymol. 482 1-33.

PETTERSEN, E.F.;GODDARD, T.D.; HUANG, C.C.; COUCH, G.S.; GREENBLATT, D.M.; MENG, E.C.; FERRIN, T.E. (2004) UCSF Chimera-A visualization system for exploratory research and analysis. J. Computational Chemistry 25, 1605-1612.

SAGHI, Z.; HOLLAND, D. J.; LEARY, R.; FALQUI, A.; BERTONI, G.; SEDERMAN, A.J.; GLADDEN, L.F.; MIDGLEY, P.A. (2011) Three-Dimensional Morphology of Iron Oxide Nanoparticles with Reactive Concave Surfaces. A Compressed Sensing-Electron Tomography (CS-ET) Approach. Nano Lett 11, 4666-4673.

SAGHI, Z.; DIVITINI, G.; WINTER, B.; LEARY, R.; SPIECKER, E.; DUCATI, C.; MIDGLEY, P.A. (2016) Compressed sensing electron tomography of needle-shaped biological 
specimens - Potential for improved reconstruction fidelity with reduced dose. Ultramicroscopy 160, 230-238.

SCHMID, G.; ZEITVOGEL, F.; HAO, L.; INGINO, P.; KUERNER, W.; DYNES, J.J., KARUNAKARAN, C.; WANG, J.; LU, Y.; AYERS, T.; SCHIETINGER, C.; HITCHCOCK, A.P.; OBST, M. (2014) Synchrotron-based chemical nano-tomography of microbial cell-mineral aggregates in their natural, hydrated state. Microsc Microanal, 20. 531-536.

SCHMID, G.; OBST, M.; WU, J.; HITCHCOCK, A.P. (2016) 3D chemical imaging of nanoscale biological, environmental and synthetic materials by soft X-ray spectro-tomography., Chapter 2 in X-ray and Neutron Techniques for Nanomaterials Characterization, C.S.S.R. Kumar, ed. 43 - 94 (Springer, Berlin)

SCHNEIDER, C.A.; RASBAND, W.S.; ELICEIRI, K.W. (2012) NIH Image to ImageJ: 25 years of image analysis. Nature Methods. 9, 671-675.

SCHRLAU, M.G.; FALLS, E.M.; ZIOBER, B.L.; BAU, H.H. (2008) Carbon nanopipettes for cell probes and intracellular injection. Nanotechnology 19, 015101.

SIDKY, E.Y.; PAN, X.C. (2008) Image reconstruction in circular cone-beam computed tomography by constrained, total-variation minimization. Physics in Medicine and Biology 53, 4777-4807.

STÖHR, J. (1992) NEXAFS Spectroscopy. (Berlin: Springer-Verlag).

SUSAC, D.; BEREJNOV, V.; HITCHCOCK, A.P.; STUMPER, J. (2011) STXM Study of the Ionomer Distribution in the PEM Fuel Cell Catalyst Layers. ECS Transactions 41, 629-635.

SUSAC, D.; BEREJNOV, V.; HITCHCOCK, A.P.; STUMPER, J. (2013) STXM

Characterization of PEM Fuel Cell Catalyst Layers. ECS Transactions 50, 405-413. 
TORRUELLA, P.; ARENAL, R.; DE LA PEÑA, F.; SAGHI, S.; YEDRA, L.; ELJARRAT, A.; LÓPEZ-CONESA, L.; ESTRADER, M.; LÓPEZ-ORTEGA, A.; SALAZAR-ALVAREZ, G.; NOGUÉS, J.; DUCATI, C.; MIDGLEY, P.A.; PEIRÓ, F.; ESTRADÉ, S (2016) 3D

Visualization of the Iron Oxidation State in FeO/Fe3O4 Core-Shell Nanocubes from Electron Energy Loss Tomography. Nano Lett., 16, 5068-5073.

VAINSHTEIN, B.K. (1970) Finding the structure of objects from projections, Sov. Phys .Crystallogr., 15, 781-787

WANG, C.; MAO, Z.; BAO, F.; LI, X.; XIE, X. (2005) Development and performance of 5 $\mathrm{kW}$ proton exchange membrane fuel cell stationary power system. Int. J. Hydrogen Energy 30, 1031-1034.

WANG, J.; HITCHCOCK, A.P.; KARUNAKARAN, C.; PRANGE, A.; FRANZ, B.;

HARKNESS, T. ; LU, Y.; OBST, M.; HORMES, J. (2011) 3D Chemical and Elemental Imaging by STXM Spectrotomography. AIP Conference Proceedings, 1365, 215-218.

WANG, J.; MORIN, C.; LI, L.; HITCHCOCK, A.P.; SCHOLL, A.; DORAN, A. (2009a) Radiation damage in soft X-ray microscopy. J. Electron Spectroscopy and Related Phenomena, 170, 25-36.

WANG, J.; BOTTON, G.A.; WEST, M.M.; HITCHCOCK, A.P. (2009b) Quantitative Evaluation of Radiation Damage to Polyethylene Terephthalate by Soft X-rays and High-energy Electrons. J. Physical Chemistry B, 113, 1869-1876.

XU, M.; WANG, L.V. (2005) Universal back-projection algorithm for photoacoustic computed tomography. Physical Review E, 71, 016706.

ZHANG, X.; BALHORN, R.; MAZRIMAS, J. (1996) Mapping and measuring DNA to protein ratios in mammalian sperm head by XANES imaging. J. Structural Biology, 116, 335-44. 
Table 1 Reconstruction Parameters used for SIRT and CS analysis of the three data sets for different numbers of angles or angle ranges

\begin{tabular}{|c|c|c|c|c|c|c|c|c|c|c|}
\hline \multirow{2}{*}{\multicolumn{2}{|c|}{$\begin{array}{c}\text { parameters } \\
\text { \# of energies }\end{array}$}} & \multicolumn{3}{|c|}{ Au nanoparticle sample } & \multicolumn{3}{|c|}{ PEM-FC model sample } & \multicolumn{3}{|c|}{ PS-acrylate sample } \\
\hline & & \multicolumn{3}{|c|}{1} & \multicolumn{3}{|c|}{4} & \multicolumn{3}{|c|}{23} \\
\hline \multicolumn{2}{|c|}{ \# of angles / range } & 67 & 23 & 12 & 37 & 19 & 10 & $\pm 90^{\circ}$ & $\pm 74^{\circ}$ & $\pm 62^{\circ}$ \\
\hline SIRT & iteration $\mathrm{N}$ & 20 & 20 & 20 & 20 & 20 & 20 & 20 & 20 & 20 \\
\hline \multirow{2}{*}{$\mathrm{CS}$} & beta & 0.7 & 0.4 & 0.2 & 0.3 & 0.1 & 0.06 & 0.2 & 0.08 & 0.05 \\
\hline & iteration $\mathrm{N}$ & 100 & 100 & 100 & 100 & 100 & 100 & 30 & 30 & 30 \\
\hline
\end{tabular}




\section{Figure Captions}

Figure 1 Schematic illustration of the compressed sensing tomographic reconstruction procedure. (a) A transform domain is selected that represents the object in a sparse way (e.g., gradient domain sparsity shown). (b) Tilt series projection data is acquired and (c) an initial reconstruction is performed by back projection or other methods. This intermediate reconstruction is likewise transformed, exhibiting less sparsity as the under-sampling artefacts are spread in a noise-like manner throughout the sparse domain. The transformed reconstruction and re-projections are refined by an iterative optimization algorithm to yield (d) the final reconstruction with (e) high sparsity in the transform domain.

Figure 2 WBP (left), SIRT (middle) and CS (right) electron tomography reconstructions of a Au nanoparticle sample. Selected slices through the $x y$ plane of (a) WBP, (b) SIRT and (c) CS reconstructions using all 67 acquired projections. Slices through the $x z$ plane are compared for three different tilt increments: (d-f) reconstructions using all 67 acquired projections, (g-i) reconstructions using 23 projections, (j-1) reconstructions using 12 projections. The insets highlight the particles near the center of the volume. See Supplemental Figure S-2 for comparison of the original $2 \mathrm{D}$ projection image at $0^{\circ}$ with that generated from the SIRT and CS reconstructions.

Figure 3 Line intensity profiles through $x z$ planes for (a) WBP, (b) SIRT, (c) CS tomographic reconstructions using 67, 23, and 12 projections as in Figure 2. The insets show the locations of the selected lines.

Figure 4 (a) C 1s and (b) F 1s spectra of the components of a model system consisting of a two layer structure in which one layer consists of $\mathrm{Pt}$ decorated graphitic carbon fibers embedded in epoxy and the second layer consists of Teflon ${ }^{\mathrm{TM}}$ fibers embedded in epoxy. The sample is a microtomed slice perpendicular to the plane of the two layers. Quantitative 2D projection maps of (c) C-fiber derived from the C 1s image sequence; (d) Pt derived from the $\mathrm{C} 1 \mathrm{~s}$ and $\mathrm{F} 1 \mathrm{~s}$ image sequences and (e) Teflon ${ }^{\mathrm{TM}}$ fiber derived from the $\mathrm{F} 1 \mathrm{~s}$ image sequence. The gray scale in (c-e) is thicknesses in nm. (f) Rescaled color composite (C-fiber in red, Teflon ${ }^{\mathrm{TM}}$ fiber in green, 
Pt in blue). The black regions in the component maps and color composite are regions filled with epoxy.

Figure 5 Slices through $x y$ and $x z$ planes of 3D volumes of the C-fiber component reconstructed by (a-c) WBP, (d-f) SIRT, and (g-i) CS-TV methods. The reconstructions were performed using $(\mathrm{a}, \mathrm{d}, \mathrm{g})$ all 37 tilt angles, (b,e,h) 19 tilt angles, and (c,f,i) 10 tilt angles. The insets highlight a selected sub-region intersected by both the $x y$ and $x z$ planes as shown by the white dashed line. (j-1) the line profile of each row. The upper images of each set is the A-B line for the xy slice, the lower images of each set is the C-D line for the $\mathrm{xz}$ slice.

Figure 6 Isosurface renderings of C-fiber (red), Teflon ${ }^{\mathrm{TM}}$ (green), and Pt (blue) components. The surfaces were determined by Otsu automatic thresholding (Otsu 1975) of the reconstruction volumes and are shown at $0^{\circ}$ (left) and $90^{\circ}$ (right) for (a-c) CS-TV reconstructions, (d-f) SIRT reconstructions and (g-i) WBP reconstructions. The reconstructions were performed using $(\mathrm{a}, \mathrm{d}, \mathrm{g})$ 37 projections, (b,e,h) 19 projections, and (c,f,i) 10 projections.

Figure 7 Effect of different choices of the $\beta$ parameter for CS-TV tomographic reconstructions. Slices through the $x y$ (upper) and $x z$ (middle) planes are shown for reconstructions using $(\mathrm{a}, \mathrm{d}) \beta$ $=0.01,(\mathrm{~b}, \mathrm{e}) \beta=0.1$, and $(\mathrm{c}, \mathrm{f}) \beta=1 .(\mathrm{g})$ line intensity profiles through the indicated line on the $x y$ slices. (h) line intensity profiles through the indicated line on the $x z$ slices.

Figure 8 (a) C 1s spectra of the components of a sample consisting of acrylate filled polystyrene microspheres inside a carbon nanopipette. Quantitative 2D projection components maps (thicknesses in nm) of (b) acrylate, (c) polystyrene (PS) microsphere shell, and (d) the carbon nanotube pipette. (e) Color-coded composite map showing acrylate in green, PS in blue, and the carbon nanotube pipette in red.

Figure 9 Evaluation of the effect of the size of the missing wedge on SIRT and CS-TV tomographic reconstructions of the acrylate maps. Comparisons of (a-c) WBP, (d-f) SIRT and (g-i) CS-TV results for three different tilt angle ranges (a,d,g) $\pm 90^{\circ}$, (b,e,h) $\pm 74^{\circ}$, and (c,f,i) $\pm 62^{\circ}$. Slices through the $x y$ (top) and $x z$ (bottom) planes are shown for each reconstruction. 
Figure 10 Line intensity profiles through selected $x z$ planes from (a) WBP, (b) SIRT and (c) CSTV reconstructions corresponding to the three tilt angle ranges indicated in Figure 9. The insets show the location of the selected lines. 